\title{
The overall design and full-aircraft aerodynamic simulation of the tilting rotor cargo UAV based on variable shaft angle gear drive
}

\author{
Chia-kan Chang ${ }^{1}$, Huang Jingjing ${ }^{1}$, Cong Zhang ${ }^{2}$, Yue $\mathrm{Gao}^{3}$, Ya-ni Wang ${ }^{4}$, E Shuang ${ }^{5}$, Chen Xubing ${ }^{2}$ \\ ${ }^{1}$ Wuhan Institute of Technology, School of Photoelectric Information and Energy Power, 430000 Wuhan, China \\ ${ }^{2}$ Wuhan Institute of Technology, School of Mechanical \& Electrical Engineering, 430000 Wuhan, China \\ ${ }^{3}$ Zhongnan University of Economics and Law, School of statistics and mathematics, 430000 Wuhan, China \\ ${ }^{4}$ Hunan University, School of Design, 410082 Changsha,China \\ ${ }^{5}$ Wuhan Institute of Technology, School of Law and Business, 430000 Wuhan, China
}

\begin{abstract}
Traditional tilt rotor aircraft rely on turning the whole engine nacelle, to accomplish the transformation between vertical take-off and landing as well as flying. This scheme has a large rotational inertia and requires a heavy and complex tilting mechanism. the engine jet flow in the process of vertical take-off and landing directly spray down, and this flow could blow up the sand and stone, let engine suck it in or hurt people. So this paper designed a new rotated way based on variable shaft Angle gear transmission. The carrier aircraft design scheme referenced the data come from following software and website. The data of airfoils come from the software profili and the website Airfoil Investigation Database. The pressure distribution as well as air flow simulation and calculation is based on XFLR5. The mechanical, appearance and overall design is based on CATIA. The gear contour design is based on CAXA CAD and the fullaircraft aerodynamic simulation is based on ANSYS/FLUENT. The engine with this design is fixed, so it only needs rotate the tilting rotor shaft, and the mechanical structure is relatively simple, which make this aircraft can play an important role in the future logistics and transportation system.
\end{abstract}

\section{Introduction}

With the progress of electronic technology and aviation theory, Industrial UAVs began to show a blowout development, the function of UAVs from folk entertainment and military spy to integrated with aerial photography, aerial surveying, short-distance delivery, reconnaissance and strike, it's becoming mature. But manned cargo planes and trucks are still the main force in the field of medium-distance express transportation.Now the technology of flight control and UAV remote control is becoming more and more mature, and the technology of tilt rotor is becoming more and more perfect,This makes it possible to design the tilting rotor cargo UAV. It could use smaller venues like parking lot or small squares to land and take off like a helicopter, due to it's fixed-wing flying mode,it could load more weight and fly longer distance than a helicopter. The aviation community expects the tilt-rotor aircraft to be the main aircraft for the next 20 years $^{[1]}$.

\section{Aerodynamic design of the vehicle}

\subsection{The design of wing, aileron and wing flap}

Because of this UAV's design concept is low speed, branch line, normal configuration propeller cargo transport plane, therefore the aerofoil selected from NACA's digit Number aerofoil, CLARK and GA-2 aerofoil(family of aerofoil), finally we analyzed various data of CLARK X, CLARK V, CLARK Z, CLARK-Y $11.7 \%$ smoothed, CLARK W, CLARK K, CLARK Y, NACA 4415, NACA 6412, NACA 4418, NACA 4421, NACA CYH, CLARK YM-15 and NASA/LANGLEY LS(1)-0413 (GA(W)-2 ${ }^{[2]}$.

In consider of machining difficulty, wing lift-drag ratio $^{[3]}$ and structural safety, preliminary define the wing taper ratio of main wing is 1 , The ratio of exposed wing's wingspan and wing chord is 6 and the wing planform is straight wing or forward-swept wing, In order to determine which wing planform suit the tilting rotor cargo UAV batter, we did some aerodynamic analysis based on XFLR5, used vortex lattice method to analyzed the aerodynamic characteristic of this two wing planform, first, The dynamic viscosity of air $\mu$ is calculated by using satland's formula:

$$
\frac{\mu}{\mu_{0}}=\left(\frac{T}{288.15}\right)^{1.5} \frac{288.15+B}{T+B}
$$

Substitution the reference kinetic viscosity of air $\mu 0=1.7894 \times 10^{-5}$ and reference temperature $B=110.4 \mathrm{~K}$,

a Corresponding author: 1042474404@qq.com 
set the temperature of air $\mathrm{T}$ as $293.15 \mathrm{~K}$, get when the air temperature is $20^{\circ} \mathrm{C}(293.15 \mathrm{~K})$, dynamic viscosity $\mu$ is $17.9 \times 10^{-5} \mathrm{~Pa} \cdot \mathrm{s}$. Then calculate Reynolds number, substituting $\mu=17.9 \times 10-5 \mathrm{~Pa} \cdot \mathrm{s}$, density of air $\rho$ refer to the air density at $20^{\circ} \mathrm{C}$ about for $1.205 \mathrm{~kg} / \mathrm{m} 3$, speed $\mathrm{v}$ is $111.11 \mathrm{~m} / \mathrm{s}$, considering that the aircraft should not occupy too much space, define it's exposed wing's wing area is $10 \mathrm{~m}^{2}$, therefore the wing chord length $\mathrm{L}$ is $1.291 \mathrm{~m}$, get the wing's Reynolds number $\mathrm{Re}$ is $1.058 \times 10^{6}$, for the sake of analysis, take $1 \times 10^{6}$ for data analysis.

Due to the efficiency of NACA 4418, NACA 4421 and NACA CYH is low within the safe Angle of attack range so they are excluded from analysis range. After analyse residual aerofoil's lift coefficient at max lift-drag ratio,Stall angle, Lift coefficient at each Angle of attack among $0 \sim 6^{\circ}$, lift-drag ratio etc. We find that NACA 6412 's lift coefficient is out-standing, and lift-drag ratio in the middle, considering that the aircraft is for intercity transportation, loadability is more important than distance, thus we choose NACA6412 as main wing's aerofoil, because of the nonlinear relationship between the lift-drag ratio and the Angle of attack, after considering the lift coefficient and drag ratio, we set the installation angle as $4^{\circ}$. The left figure is the main wing use NACA 6412 as it's aerofoil under the conditions listed above, lift coefficient curves at different angles of attack, the horizontal coordinate is the Angle of attack and the vertical coordinate is the lift coefficient. The right figure is the main wing use NACA 6412 as it's aerofoil under the conditions listed above, the lift-drag ratio curve among the $0 \sim 6^{\circ}$ angle of attack, the horizontal coordinate is the Angle of attack and the vertical coordinate is the lift-drag ratio.

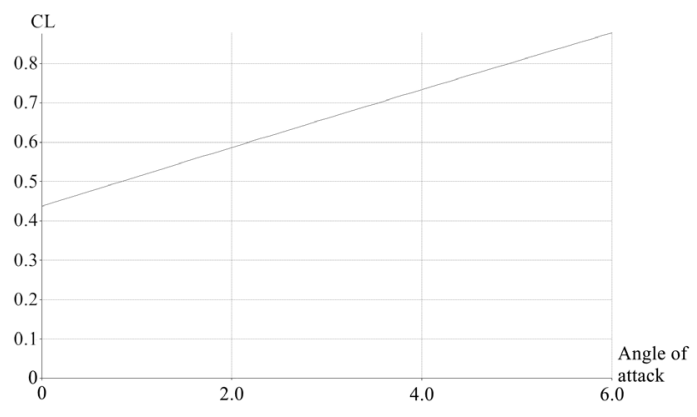

Figure 1. Statistical graph of change of the attack Angle and lift coefficient

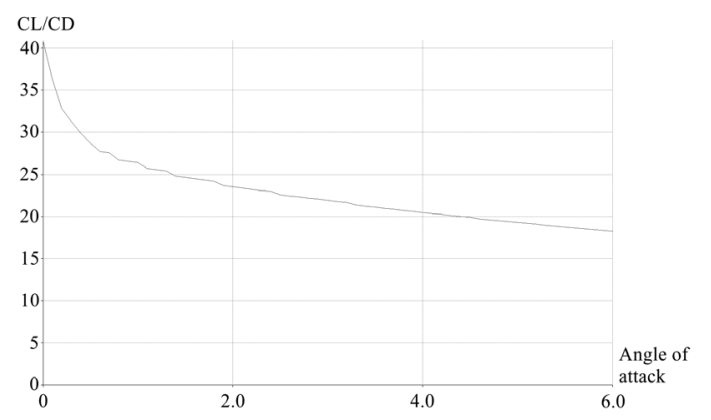

Figure 2. Statistical diagram of change of the attack Angle and lift-drag ratio
From the figures, the CL and CL/CD of the straight wing with NACA6412 aerofoil under the conditions listed above is 0.732 and 20.43 .

Then verify the influence of sweep forward Angle on lift coefficient and drag ratio under the same conditions as the aspect ratio and airfoil area, set attack angle as $4^{\circ}$, sweep forward Angle as $5^{\circ}, 10^{\circ}, 15^{\circ}, 20^{\circ}$, use XFLR5's vortex lattice method to analyse, get the table below:

Table 1. Statistical table of wing sweep forward Angle, lift coefficient and lift-drag ratio.

\begin{tabular}{|c|c|c|}
\hline sweep forward Angle & $\mathrm{CL}$ & $\mathrm{CL} / \mathrm{CD}$ \\
\hline $0^{\circ}$ & 0.732 & 20.43 \\
\hline $5^{\circ}$ & 0.731 & 20.53 \\
\hline $10^{\circ}$ & 0.727 & 20.68 \\
\hline $15^{\circ}$ & 0.718 & 21.11 \\
\hline $20^{\circ}$ & 0.706 & 21.37 \\
\hline
\end{tabular}

It can be seen that under the above conditions, the increase of forward sweep Angle will increase the liftdrag ratio and decrease the lift-drag coefficient. Moreover, with the increase of forward sweep Angle, the variation range of lift-drag ratio and lift-drag ratio will increase. However, because the forward sweep wing can reduce the lift coefficient and greatly increase the torsion force at the point where the wing connects to the fuselage in the vertical take-off and landing state, therefore we use straight wing design.

In the design of flaps, referred the various types of flap's lift section curve diagram ${ }^{[3]}$ and their polar curve diagram $^{[3]}$, finally adopt single-slotted flap design scheme, in terms of design parameters, the chord length is tentatively fixed at local wing chord length's $25 \%{ }^{[3]}$, set the length of flap as 0.6 wingspan, therefore the chord length is $0.323 \mathrm{~m}$ and the length of flap is $2.32 \mathrm{~m}$. Angle of flap defection referred Aircraft design manual ${ }^{[3]}$, combined in this paper its chord length is $25 \%$ of the wing chord length and combined with the design of the aircraft wing, set the angle of flap defection as $40^{\circ}$.

According to the statistics of relative area of ailerons ${ }^{[3]}$, the aileron area of the aircraft is defined as $6 \%$ of the wing area, this value is $0.697 \mathrm{~m}^{2}$, set the length of the aileron as 0.4 wingspan as $3.098 \mathrm{~m}$, therefore aileron chord length supposed to be $0.225 \mathrm{~m}$.

Some data of the above design are determined by calculation and reference to other literature, it can be fine-tuned or modified by wind tunnel test.

\subsection{Empennage design}

\subsubsection{Vertical tail and rudder design}

According to the formula:

$$
S_{V T}=\frac{C_{V T} b_{w} S_{w}}{L_{V T}}
$$


estimate vertical tail area $S_{V T}$, set the the length of the inside cross-section of the fuselage as $1 \mathrm{~m} \times 1 \mathrm{~m}$, set wingspan $b_{w}$ as $9 \mathrm{~m}$, tail capacity of vertical tail $C_{V T}$ referred some propeller feeder planes ${ }^{[4]}$ and set as 0.08 , tail moment arm of vertical tail LVT set as $4.5 \mathrm{~m}$, then get the value of $S_{V T}$ is $1.856 \mathrm{~m}^{2}$. The aspect ratio of the vertical tail referred the data given in aircraft design manual $^{[3]}$, set as 1.7 , To determine the influence of wing taper ratio and sweep Angle on the air drag of vertical tail. The drag coefficient of three vertical tails with NACA 0011 aerofoil was analyzed by XFLR5's vortex lattice method, value of aspect ratio $\mathrm{A}$ is 1.7 and $\mathrm{S}_{\mathrm{VT}}$ is $1.6 \mathrm{~m}^{2}$, but different wing taper ratio and sweepback angle on leading edge:

Table 2. The parameters of three vertical tails to be tested.

\begin{tabular}{|c|c|}
\hline $\begin{array}{c}\text { wing taper } \\
\text { ratio } \lambda\end{array}$ & $\begin{array}{c}\text { sweepback } \\
\text { angle on } \\
\text { leading } \\
\text { edge }\left(^{\circ}\right)\end{array}$ \\
\hline 0.32 & 0 \\
\hline 0.32 & 35 \\
\hline 1 & 0 \\
\hline
\end{tabular}

It was found that the drag coefficient of the three tail fins was the same, for aesthetic reasons, set wing taper ratio $\lambda$ as 0.32 , sweepback angle on leading edge as $35^{\circ}$, vertical tail's MAC position of wingspan direction $\mathrm{y}_{\mathrm{cA}}$ could be calculate by the formula:

$$
y_{c A}=\frac{\sqrt{A S}}{6} \frac{1+2 \lambda}{1+\lambda}
$$

Get the value of $\mathrm{y}_{\mathrm{cA}}=0.37 \mathrm{~m}$. Install the horizontal tail on $\mathrm{y}_{\mathrm{cA}}$, make the horizontal tail's $25 \% \mathrm{MAC}$ and vertical tail's $25 \% \mathrm{MAC}$ at the same point, use symmetrical aerofoil with moderate thickness such as NACA 0011.

The area of the rudder is estimated according to the statistical curve diagram of the relation between the relative area of rudder and the total area of wing ${ }^{[3]}$ and the data of civil jet airliner in geometric data sheet for vertical tail and rudder ${ }^{[3]}$. Combine the situation of this plane, set the relative area of rudder's value as $30 \%$, $0.56 \mathrm{~m}^{2}$. Set the rudder span as $1.58 \mathrm{~m}$, divided in two parts, the rudder span of the rudder above the horizontal tail is $1.31 \mathrm{~m}$, he rudder span of the rudder below the horizontal tail is $0.27 \mathrm{~m}$. The chord length of rudder in both sides is $0.354 \mathrm{~m}$.

\subsubsection{Horizontal tail and elevator design}

In order to make the projection of the full-aircraft pressure center on the fuselage coincide with the center of gravity. Therefore, the lifting force of the wing was analyzed to determine its pressure center, after analyzed by XFLR5 we get the lift distribution of wing cross section:

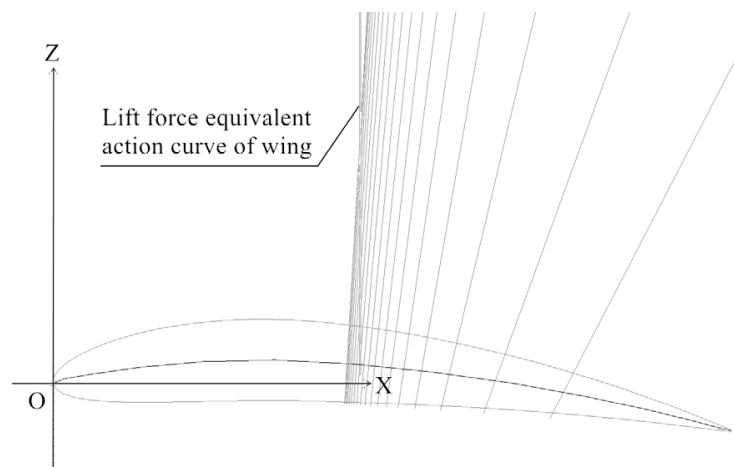

Figure 3. lift distribution of wing cross-section

The equivalent pressure center can be obtained at $45.3 \%$ of the cross section of the wing. In order to keep the aircraft in static stability, the position of the center of gravity is set before $25 \% \mathrm{MAC}$ and coincides with the center of pressure, so the position of the center of pressure needs to be adjusted by the horizontal tail. The selection of horizontal tail's aerofoil will consider the air drag and the reverse lift it provide. In order to prevent the change of the center of gravity of the full-aircraft in actual use, there is a pair of propellers for leveling under the condition of vertical take-off and landing inside the horizontal tail, in vertical take-off and landing state, the horizontal tail will slide outward, and the tail rotor is exposed, in order to facilitate the design of this mechanism and improve the horizontal tail efficiency in practical use ${ }^{[4]}$, set the horizontal tail's wing taper ratio as 1 , Because the radius of the tangential circle of the rectangle is larger than that of other parallelogram with the same area, therefore set the sweep angle of horizontal tail as $0^{\circ}$. Therefore, the aerodynamic center of fullaircraft can be determined to confirm the position of the center of gravity of full-aircraft.

As this plane is a propeller conveyor, the value of tail capacity coefficient of horizontal tail $\mathrm{V}_{\mathrm{HT}}$ refer to each propeller feeder aircraft ${ }^{[8]}$, provisional set as 1.00, according formula:

$$
S_{H T}=\frac{V_{H} T c A S_{w}}{L_{H T}}
$$

To estimated horizontal tail area $\mathrm{S}_{\mathrm{HT}}$, full area of main wings $S_{w}$ is $11.6 \mathrm{~m}^{2}$, MAC length of main wings $c_{A}$ is $1.291 \mathrm{~m}$, tail moment arm $\mathrm{L}_{\mathrm{HT}}$ estimate according to fuselage length, in order to save space, the length of the fuselage is tentatively fixed at $9 \mathrm{~m}$, the tail moment arm is estimated at $50 \%$ of the fuselage length, the value is $4.5 \mathrm{~m}$. Get the area of horizontal tail $\mathrm{S}_{\mathrm{HT}}$ as $3.328 \mathrm{~m}^{2}$.

According to this data, the aerodynamic center of the full-aircraft can be analyzed. Since the speed of the wing is far less than the critical Mach number in the flight environment, the following formula can be used:

$$
\begin{gathered}
C_{l \alpha}=\frac{1.05}{\beta}\left(\frac{C_{l \alpha}}{C_{l \alpha, \text { the }}}\right) C_{l \alpha, \text { the }} \\
C_{l \alpha, W}=\frac{2 \pi A}{2+\sqrt{\frac{A^{2}}{K^{2}}\left(\beta^{2}+\operatorname{tg}^{2} \Lambda_{1 / 2}\right)+4}}
\end{gathered}
$$




$$
\begin{gathered}
K=\frac{C_{1 \alpha}}{2 \pi} \\
\beta=\sqrt{1-M^{2}} \\
C_{l a, \text { the }}=6.28+4.7\left(\frac{t}{c}\right)(1+0.00375 \tau)
\end{gathered}
$$

Estimate the slope of lift curve of main wing, get the value of trailing edge angle $\tau$ in Airfoil Investigation Database as $21.3^{\circ}$, the value of $t / c$ is 0.12 . The value of $\operatorname{tg}[(1 / 2) \tau]$ is calculated as 0.188 , set the value of $R e$ as $10^{6}$, the value of modified empirical factor $\left(\mathrm{C}_{l \alpha} / \mathrm{C}_{l \alpha}\right.$,the $)$ could be find from the graph ${ }^{[3]}$, get the value of modified empirical factor $\left(\mathrm{C}_{\mathrm{l} \alpha} / \mathrm{C}_{l \alpha \text {,the }}\right)=0.7$, theoretical slope of lift curve of aerofoil $\mathrm{C}_{l \alpha \text {,the }}$ is calculated according to formula(9) as 6.88, substitute the mach number of $111.11 \mathrm{~m} / \mathrm{s}$ valued 0.33 into formula(8)and get the value of $\beta=0.94$, substitute it into formula(5)and get the value of lift curve of aerofoil $\mathrm{C}_{\mathrm{l \alpha}}=5.40$, substitute it into formula(7)and get the value of $\mathrm{K}=0.86$, substitute it into formula(6)and combined with the data of the wing, get the value of wing's slope of lift curve $C_{L \alpha, W}=4.257$. The following formulas could be used:

$$
\begin{gathered}
K_{W F}=1+0.025 \frac{d_{F}}{b}-0.25\left(\frac{d_{F}}{b}\right)^{2} \\
C_{L \alpha, W B}=K_{W F} C_{L \alpha, W} \\
d_{F}=\sqrt{\frac{4 S_{f, \max }}{\pi}}
\end{gathered}
$$

To calculate the value of Wing-body interference factor $K_{W F}$ and slope of lift curve of wing-body combination $\mathrm{C}_{L \alpha, \mathrm{WB}}$, set the value of cross section area of the most wide part of fuselage $\mathrm{S}_{\mathrm{f}, \max }$ as $2.020 \mathrm{~m}^{2}$, substitute it into formula(12)and get the value of $d_{F}=1.604$. Substitute the value of wingspan $b=9 m$, into formula(10) and get the value of $\mathrm{K}_{\mathrm{WF}}=0.992$, substitute it into formula(11)and combined with the calculated slope of the lift curve of the wing, get the value of $\mathrm{C}_{L \alpha, \mathrm{WB}}=4.222$. full-plane's aerodynamic center $\mathrm{X}_{\mathrm{F}}$ could be calculated by the following formulas:

$$
\begin{gathered}
\left(\frac{X_{a c}}{c_{A}}\right)_{W B}=\left(\frac{X_{a c}}{c_{A}}\right)+\frac{\Delta f_{1} X_{a c}}{c_{A}}+\frac{\Delta f_{2} X_{a c}}{c_{A}} \\
\frac{\Delta f_{1} X_{a c}}{c_{A}}=-\frac{1.8}{C_{L \alpha W B}} \frac{b_{f} h_{f} l_{f n}}{S c_{A}} \\
\frac{\Delta f_{2} X_{a c}}{c_{A}}=\frac{0.273}{1+\lambda} \frac{b_{f} c_{g}\left(b-b_{f}\right)}{c_{A}{ }^{2}\left(b+2.15 b_{f}\right)} \tan \Lambda_{1 / 4} \\
X_{F}=X_{a c}+\frac{q H T}{q} \frac{C_{L \alpha H}}{C_{L \alpha}} \frac{S_{H} l_{H}}{S c_{A}}\left(1-\frac{d \varepsilon}{d \alpha}\right)+\Delta P X_{a c}
\end{gathered}
$$

$$
\begin{gathered}
\frac{d \varepsilon}{d \alpha}=\frac{2}{\pi A_{q}} \times 57.3 C_{L \alpha W B} \\
C_{L \alpha}=C_{L \alpha W B}+C_{L \alpha H} \frac{S_{H}}{S}\left(\frac{1-\mathrm{d} \varepsilon}{d \alpha}\right) \frac{q_{H}}{q} \\
A_{q}=\frac{b^{2}}{S}
\end{gathered}
$$

Set $\mathrm{X}_{\mathrm{acW}}$ as $25 \% \mathrm{MAC}$, valued $0.323, \mathrm{~b}_{\mathrm{f}}$ and $\mathrm{h}_{\mathrm{f}}$ set as $1.554 \mathrm{~m}$ and $1.30 \mathrm{~m}, \mathrm{c}_{\mathrm{A}}$ valued $1.291 \mathrm{~m}, \mathrm{~S}$ valued $10 \mathrm{~m}^{2}$, substitute $\mathrm{C}_{L \alpha, \mathrm{WB}}=4.222$ into formula(14)and get the correction $\Delta \mathrm{f}_{1} \mathrm{X}_{\mathrm{ac}}$ 's value as $-0.086 \mathrm{l}_{\mathrm{fn}}$, aerodynamic center of wing-body combination $X_{a c}$ valued 0.323 $0.0861_{\text {fn }}$. Because of the plane's horizontal tail is installed at the middle of the vertical tail, so the value of $\mathrm{q}_{\mathrm{HT}} / \mathrm{q}^{[5]}$ is 0.95 , wingspan $b=9 \mathrm{~m}$, combine the known conditions and simultaneous formula(17)(18)(19)(16), get the value of full-plane's aerodynamic center $X_{F}$ as $4.5 \frac{C_{L \alpha H} l_{H}}{5.018 C_{L \alpha H}+4.31}+0.323-0.0861_{f n}+\Delta P X_{a c}$

The following formula is used to modify the effect of the propeller on the aerodynamic center:

$$
\Delta P \frac{X_{a c}}{c_{A}}=-0.05 \sum \frac{D_{P}{ }^{2} B_{P} l_{P}}{S c_{A} C_{L \alpha W B}}
$$

Diameter of propeller $\mathrm{D}_{\mathrm{P}}$ set as $7 \mathrm{~m}$, propeller Blade Number $B_{P}$ set as $3, l_{P}$ set as $0.923 \mathrm{~m}$, The influence of the propeller on the aerodynamic center can be obtained as -0.249 , set the aspect ratio of the horizontal tail as 3.4, then it can be calculated according to the known conditions that $\mathrm{l}_{\mathrm{H}}=0.989 \mathrm{~m}, \mathrm{l}_{\mathrm{fn}}=3.317 \mathrm{~m}$.

The formula can be obtained after simplification:

$$
X_{F}=4.45 \frac{C_{L \alpha H}}{5.018 C_{L \alpha H}+4.31}-0.284
$$

Adopt NACA 0013 as the aerofoil of horizontal tail to verify the feasibility of the design, using the formula $(5)(6)(7)(8)(9)$, and use the same method with calculating the slope of lift curve of wing to calculate the slope of lift curve of horizontal tail, It is found that the aerodynamic center of full-aircraft always before the pressure center, the aerodynamic center located at $32 \% \mathrm{MAC}$ but the pressure center located at $45 \% \mathrm{MAC}$, if the center of pressure coincides with the center of gravity, in order to ensure flight safety, the after limit of $\mathrm{cg}$ should be at the front of the aerodynamic center for at lest $3 \% \mathrm{MAC}^{[5]}$, therefore, the horizontal tail is required to provide reverse lift to adjust the position of pressure center.The density of air $\rho$ set as $1.205 \mathrm{~kg} / \mathrm{m}^{3}$, velocity $\mathrm{V}$ is $111.11 \mathrm{~m} / \mathrm{s}$, aspect ratio $A_{w}$ is 3.4 , chord length of horizontal tail is $0.989 \mathrm{~m}$, attack angle set as $0^{\circ}$, wing planform set as straight wing. Use XFLR5's vortex lattice method to analyse the horizontal tail with high lift-drag ratio aerofoil such as NACA 65-410, NACA 0010-34 a=0.8 $\mathrm{c}(\mathrm{li})=0.2$, NACA 63-412, NACA 63(2)615, NACA 65(1)-412, NACA 2.5411, NASA/LANGLEY LS(1)-0413 (GA(W)-2), NACA 
4415, NACA 63A210, CLARK K ${ }^{[2]}$ etc. The parameter of horizontal tail has enumerated above, analyse the lift force and liftdrag ratio of the horizontal tail with different aerofoil and it is calculated that if the horizontal tail's aerofoil is NACA 0013, the lift curve slope is 3.532 , taking the front $3 \% \mathrm{MAC}$ of the aerodynamic center as the afterward limit of center of gravity, the reverse lift needs to be provided more than 2930.391N, which is a reference. Considering the lift-drag ratio of the horizontal tail and the reasonable range of lift, use horizontal tail with reverse NACA 63-412 aerofoil to calculate, it is known that the aerodynamic center of fullaircraft is in $32 \% \mathrm{MAC}$, and the pressure center is located in the $18 \% \mathrm{MAC}$. When the center of gravity is designed near the pressure center, the center of gravity is about $14 \%$ MAC before the aerodynamic center, satisfies the design requirements.

After check the Statistical chart of relative area of elevator ${ }^{[3]}$ the area of the elevator is estimated to be $32 \%$ of the area of the horizontal tail, $0.918 \mathrm{~m}^{2}$, set the span of the elevator as $3 \mathrm{~m}$, then the length of the elevator is $0.306 \mathrm{~m}$.

The afterward limit of center of gravity is located at the $3 \% \mathrm{MAC}$ front of the aerodynamic center of fullaircraft, which is $29 \% \mathrm{MAC}$. The forward limit of center of gravity requires further aerodynamic simulation of the full-aircraft before it can be determined.

\subsection{Fuselage design}

As the plane is a logistics UAV, the cross section of the inner warehouse is set to a square of $1 \mathrm{~m}^{2}$, Since there is no pressurize in the body, the wall thickness set as $5 \mathrm{~cm}^{[4]}$, the length / diameter ratio of the front and rear fuselage is set to 2 and $3.0^{[4]}$, according to fuselage diameter and the length of the front and rear fuselage set as $2.482 \mathrm{~m}$ and $3.724 \mathrm{~m}$. The tail contraction angle $\beta_{\mathrm{F}}$ and rear body upswept angle $\psi$ are both 12 degrees. The shape of the nose is obtained with parabolic rotation.

\section{Design of mechanical structural of key parts of aircraft and engine nacelle}

\subsection{Design of tilt rotor mechanical structure and engine nacelle}

\subsubsection{Design of engine inlet port and engine nacelle shape}

The engine is designed to adopt a turboprop engine with a variable pitch propeller, because the design needs to ensure that the aircraft can rise vertically at the speed of $10 \mathrm{~m} / \mathrm{s}$ at the maximum load, the power of a single engine must be more than $275 \mathrm{~kW}$, and the engine intake is designed by the standard of PT6A-15AG turboprop engine of Pratt \& Whitney of Canada. The tilt system of the rotor is designed to use the tilting mechanism based on the turbine worm. The area of the air inlet could be calculated according following formulas:

$$
\begin{gathered}
S_{\text {intake }}=\frac{m_{\text {air }}}{v_{\text {intake }} \rho_{\text {intake }}} \\
v_{\text {intake }}=-v_{0}
\end{gathered}
$$

The specific heat ratio $\gamma$ is calculated as 1.40 according to the basic relationship of one-dimensional steady adiabatic flow, when the Mach number is 0.32 , the value of $\rho_{\text {intake }}$ is $1.145 \mathrm{~kg} / \mathrm{m}^{3}$, when the value of $\overline{\mathrm{V}}$ is 0.5 , get the value of $v_{\text {intake }}$ as $55.556 \mathrm{~m} / \mathrm{s}$, find the engine air flow $\mathrm{m}_{\text {air }}$ of PT6A-15AG is $2.8 \mathrm{~kg} / \mathrm{s}$, substitute it into formula(22), the section area of air inlet is calculated as $0.044 \mathrm{~m}^{2}$.

In the preliminary design of the shape of inlet lip, single-spoon intake port ${ }^{[6]}$ design is adopted, the lip crown is thinner because of the different airflow status in different area, the keel of lip is thicker and the lateral lip is in a medium thickness ${ }^{[7]}$, The contraction ratio of the lip crown is $25 \%$, the lateral lip is $28 \%$, the keel of lip is $30 \%$. The ratio of the throat area and the inlet area $\mathrm{A}_{\mathrm{i}} / \mathrm{A}_{\mathrm{m}}$ is $52.2 \%$. The specific parameters are shown in the diagram:

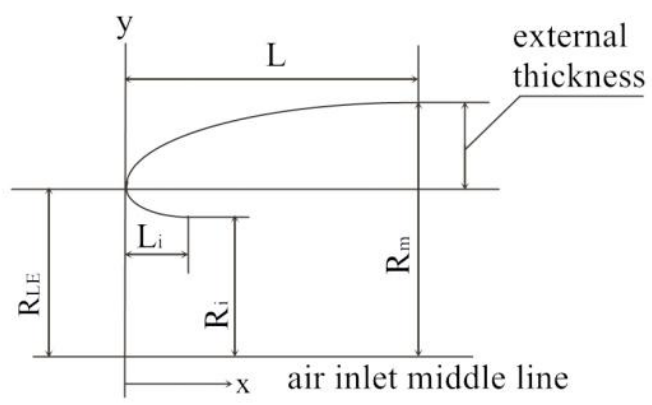

Figure 4. Lip parameters of the air inlet

Define formula:

$$
K_{1}=\frac{R_{L E}}{R_{i}}
$$

$$
\frac{R_{L E}}{R_{m}}=K_{1} \sqrt{\frac{A_{i}}{A_{m}}}
$$

$$
\frac{R_{m}}{L}=K_{2}+K_{3}\left(\frac{A_{i}}{A_{m}}\right)^{3}
$$

$$
\frac{R_{i}}{L_{i}}=\frac{1}{K_{4}\left(K_{1}-1\right)}
$$

Calculated $\mathrm{K}_{1}$ of top of lip, lateral lip and lip ridge are respectively $1.14,1.16$, and 1.18 . The value of $K_{2}$ is set as 0.2 , the value of $K_{3}$ is set as 12.5 , and the value of $\mathrm{K}_{4}$ is set as $1.5^{[8]}$. Due to the design of variable shaft angle gear drive mode, the whole engine nacelle is not required to be tilted. Therefore, the angle of attack of the air inlet caused by the change of tilt angle during engine nacelle tilt is not considered. Therefore, the preliminary design of the lip of the inlet can be obtained directly. The 
details need further wind tunnel test to complete the optimization. This article will not continue to go deep.

According to the length and diameter of the engine, as well as the volume set aside for the tilting mechanism and the reduction gear box, the width of the engine nacelle is set as $500 \mathrm{~mm}$, and the height is set as $800 \mathrm{~mm}$, and the length is set as $2600 \mathrm{~mm}$. Refer to the air inlet shape design of planes use PT6A as it's engine like King Air C90, The original design of the air inlet width is $400 \mathrm{~mm}$, the side lip is two semicircle, and the inlet height is calculated to be $117.394 \mathrm{~mm}$. Taking the volume of the tilting mechanism into account, a preliminary design plan for the nacelle configuration of the engine can be obtained. The red part is covered with soft skin. This part can be folded when the rotor is tilted, won't affect the normal tilt of the rotor, and will be strained in the fixed wing flight mode. The appearance, as shown in diagram 6 , does not affect the aerodynamic performance. As the specific models of the reduction gear box and the type of stepper motor used to drive the gearbox are not determined, the specific appearance may be modified on this design. The following figures is the design of the engine nacelle and the structure beneath the skin:

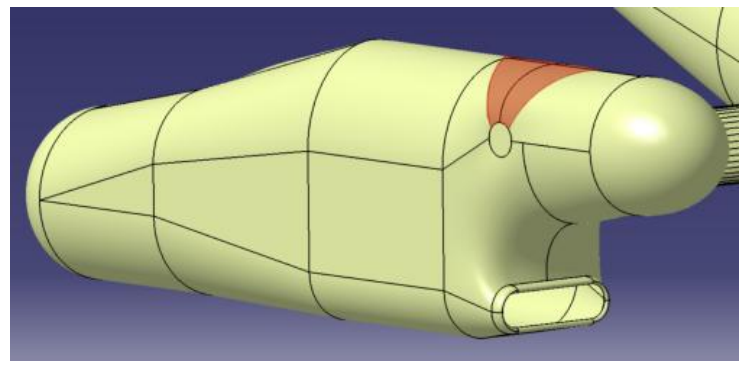

Figure 5. engine nacelle

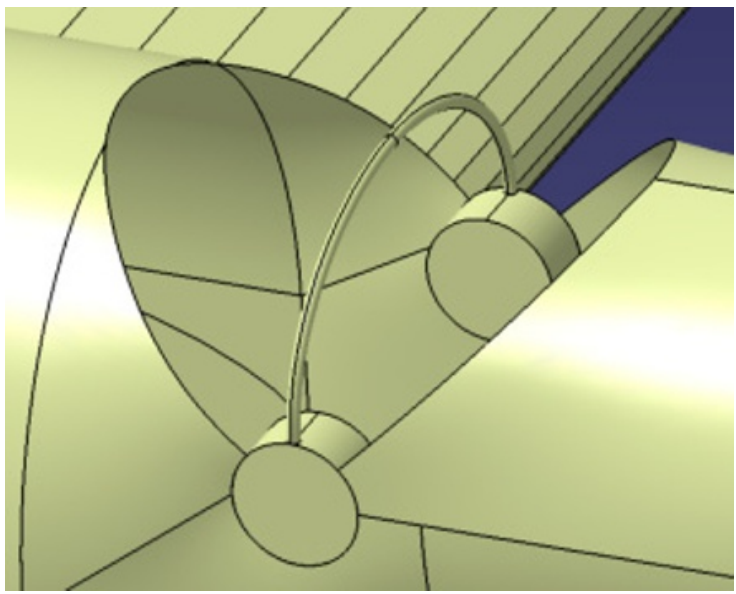

Figure 6. structure under the soft skin

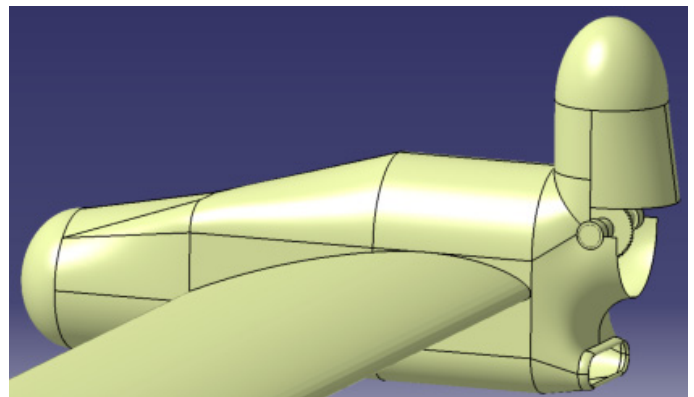

Figure 7. engine nacelle in Vertical take-off and landing mode

\subsection{2 the design of Mechanical structure used in rotor tilting}

The rotor tilting mechanism use the transmission with variable shaft angle mode based on bevel gear group, using a set of bevel gear as the core of the mechanism, The specific design is shown in the following figures:

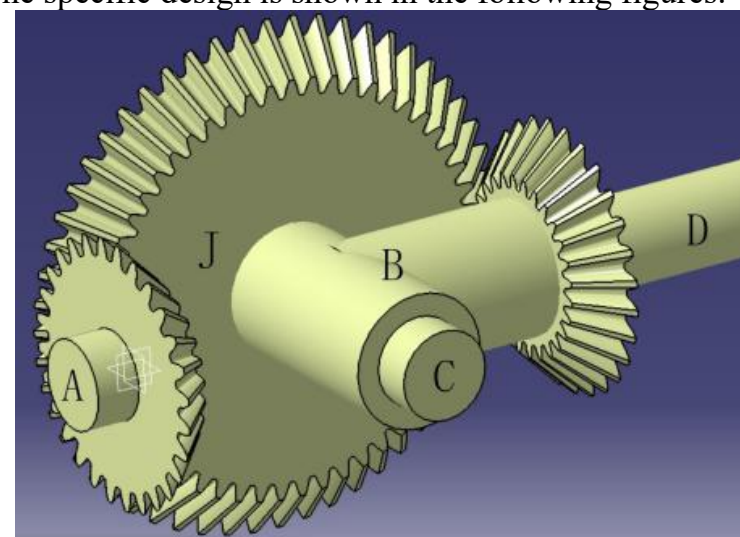

Figure 8. State of the tilting mechanism of the fixed wing mode

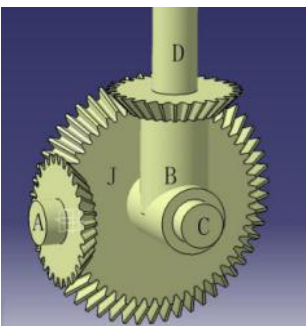

Figure 9. State of the tilting mechanism of vertical take-off and landing mode

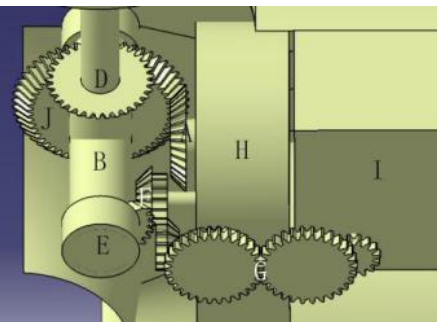

Figure 10.installation(vertical take-off and landing mode)
The $\mathrm{A}$ is the gear and shaft connecting the engine deceleration gear group $\mathrm{H}$; B is a shaft sleeve, within a rotating shaft $\mathrm{C}$ connected with the gear $\mathrm{J}$ and gear $\mathrm{D}$ with the rotor connect with it. B is controlled by the tilting gear group $\mathrm{F}$, and it can achieve the mutual conversion between Figure 7 and figure 8. Gear D and the rotating shaft connected to it deal with power output, connecting to the propeller; $\mathrm{E}$ is the connecting mechanism between the tilting mechanism and the engine nacelle shell; $\mathrm{G}$ is the gear group connecting the deceleration gear box I which connect large torque stepper motor which provide the power needed for tilting and tilting gear group F.

\subsection{Internal structure design of empennage}

Due to the rotor use variable pitch propeller which do not have flapping motion, a set of propellers is needed to assist the balance in the empennage, to ensure the stability of the aircraft in the state of vertical take-off and landing. In order to prevent the effect of the tail rotor propeller on the overall aerodynamic performance, the empennage propeller is installed inside the empennage, and will be used only in the vertical take-off and landing state. The specific design scheme is shown in the following figures: 


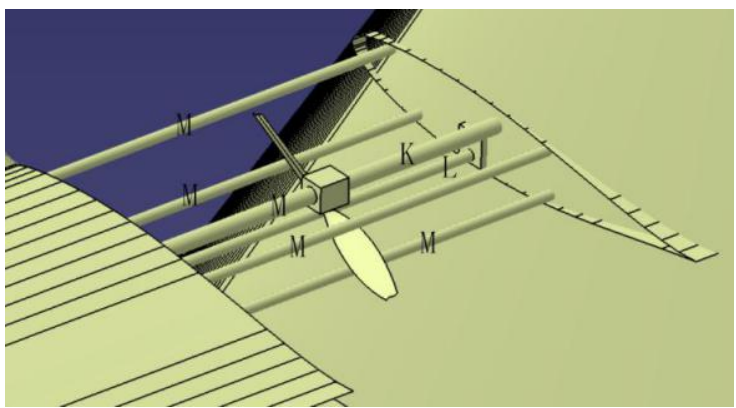

Figure 11. empennage structure in state of vertical take-off and landing mode

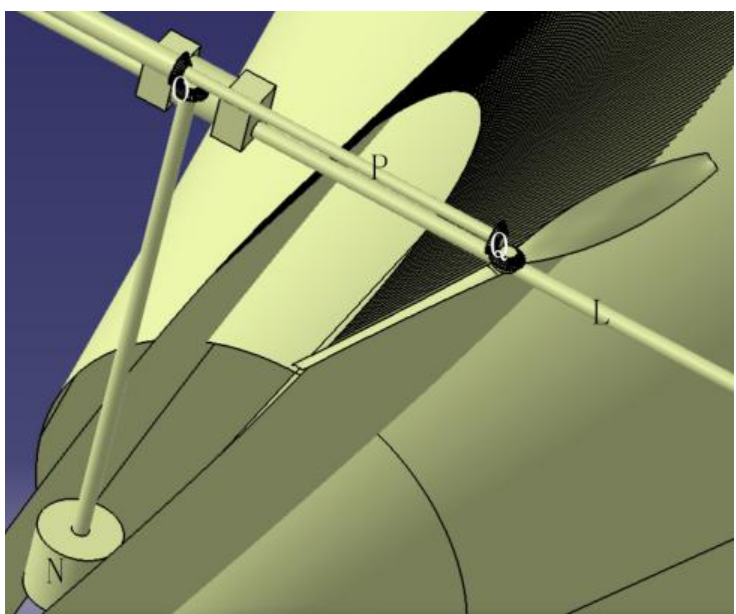

Figure 12. core structure of the internal empennage

$\mathrm{K}$ is a shaft sleeve, contain a driving shaft $\mathrm{P}$ that drives the propeller of the empennage; $\mathrm{L}$ is a rotating shaft with a thread, which is connected to a double out shaft motor based on two turbine worm self-locking mechanisms and controls the flex movement of horizontal empennage; $M$ are five smooth shafts with the function of fixing and could embed signal lines in them. $\mathrm{N}$ contains the brushless electric motor which provide power for two empennage propellers and it's transmission shaft. $\mathrm{O}$ is a bevel gear group that drives the P shaft; Q is another bevel gear group, which is used to transfer the power of $\mathrm{P}$ shaft to the empennage propeller. This device can ensure that two rotor propellers have the opposite rotation direction and the same rotation speed, and compare the scheme of one motor driving a propeller, this scheme can avoid the situation that one of the motor failures leading to an accident of the aircraft thus improve its reliability.

\subsection{Design of take-off and landing system}

As the aircraft is a vertical take-off and landing aircraft, it does not consider the situation of sliding take-off and landing. In order to reduce the air drag in the cruise state, so it is proposed to use the retractable wheeled landing gear or retractable skid landing gear, for the convenience of ground staff to move the aircraft, so we intends to use the wheel type landing gear based on a universal wheel with a rotating shaft which can be locked.

Because there is no need to take the situation of takeoff and landing into account, so we ignored the situation like aircraft tail rubbing ground when defining the landing gear layout, The UAV landing gear load standard is designed according to the standard of general purpose aeroplane, need to make sure the landing gear can handle $3 \mathrm{~g}$ load ${ }^{[9]}$, this paper designed a tricycle landing gear for this $\mathrm{UAV}$, the installation position in a side view is shown in the figure:

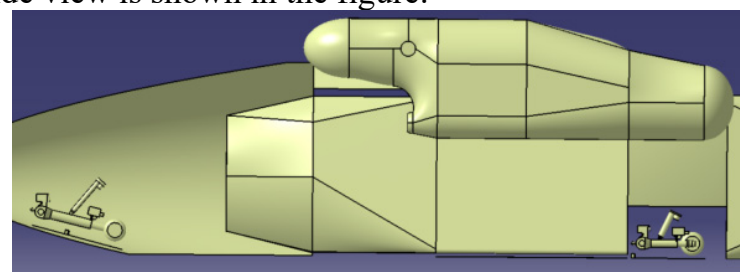

Figure 13. The installation position of the landing gear in a side view

Specific mechanical structure of the front and rear landing gear:

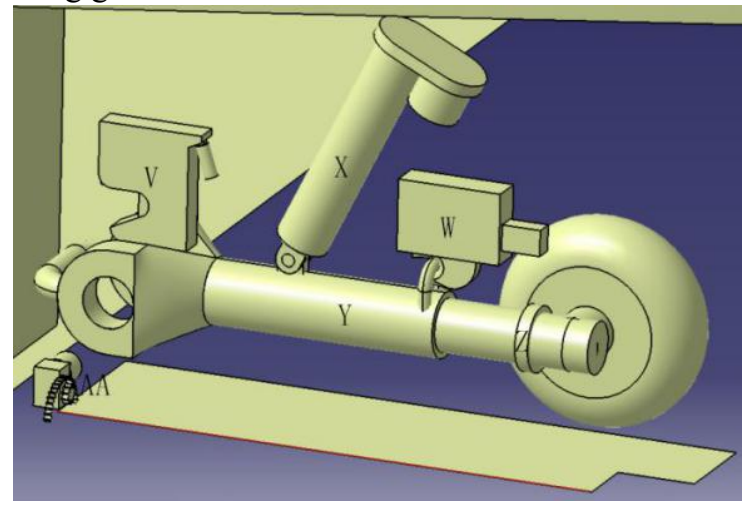

Figure 14. Structure of rear landing gear

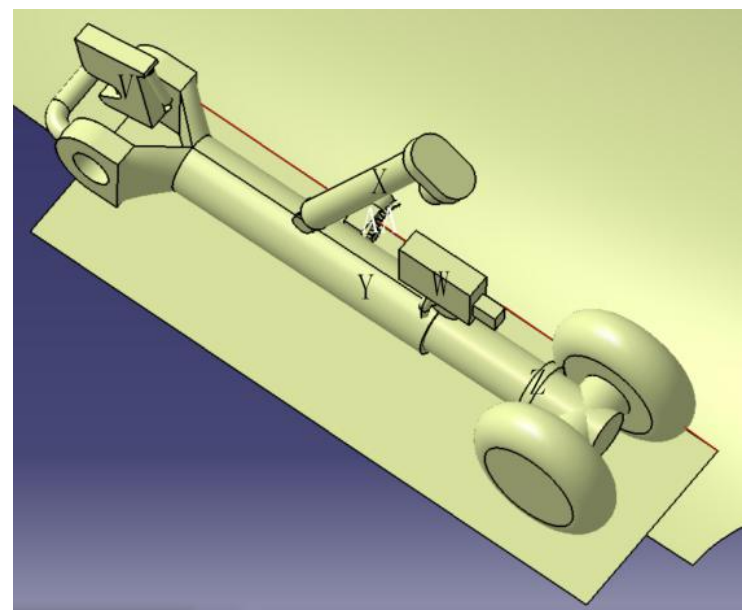

Figure 15. Mechanical structure of front landing gear

$\mathrm{X}$ is the landing-gear retraction jack based on electric drive pusher. $\mathrm{V}$ is the down lock of landing gear, which is to prevent the impact and pressure to $\mathrm{X}$ when plane is landing or on the ground. $\mathrm{W}$ is the gear uplock, which locks automatically when the landing gear is closed. After being locked, $\mathrm{X}$ can cut off the power to reduce the working time of $\mathrm{X}$ and increase its service life. $\mathrm{Y}$ is the main body and buffer of the landing gear, using oleopneumatic buffer. $Z$ is a universal shaft that can be locked physically by manual. It allows the ground staff to move the aircraft after landing. AA is the switching mechanism of landing gear door, which is composed of a motor with a worm gear deceleration and a set of gear groups that control the door opening and closing, so that 
the hatch door can be rotated along the axis shown in the red line.

$\mathrm{V}$ and $\mathrm{W}$ are hook ring locks, and the internal structure is shown in the following diagrams:
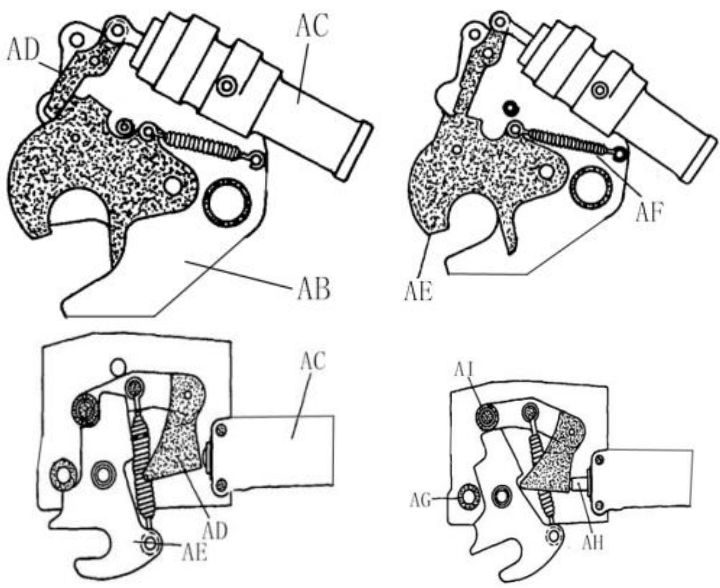

The four diagrams are structures of the uplock in unlocking condition, the uplock in locking condition, the downlock in locking condition and the downlock in unlocking condition. The $\mathrm{AB}$ is the side plate, the $\mathrm{AC}$ is the actuating jack, $\mathrm{AD}$ is the locking key, $\mathrm{AE}$ is the lock hook, AF is the mechanical spring, AG is the stopping platform, $\mathrm{AH}$ is the piston rod and $\mathrm{AI}$ is a roller.

\section{Aerodynamics simulation of full- aircraft}

Based on the current aerodynamic shape design, we import it into Designmodeler module and set a Enclosure for it as it's simulated wind-tunnel wall:

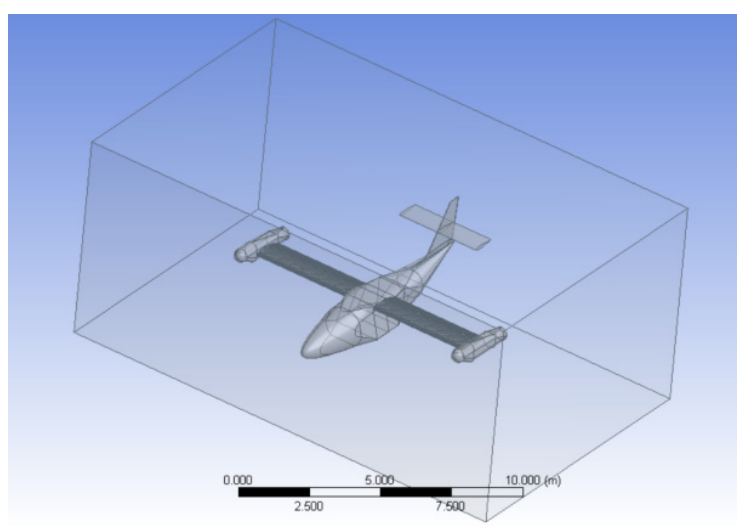

Figure 16. plane and the Enclosure

Then import it into Mesh module to divide the grid and name the simulated wind tunnel wall as 'inlet', then update it and import it into ANSYS/FLUENT, use the density-based solver with SST k-moega viscous model, set the boundary conditions of inlet as Pressure Far-Filed, under the condition of ideal gas, the flow is taken in the increasing $\mathrm{x}$ direction (the direction right to the nose) at the speed of $0.3 \mathrm{M}$, and the aerodynamic simulation is carried out at the atmospheric pressure of the 95200pa (the atmospheric pressure at the altitude of $500 \mathrm{~m}$ ), plus, the attack angle of the aircraft is 0 degree,

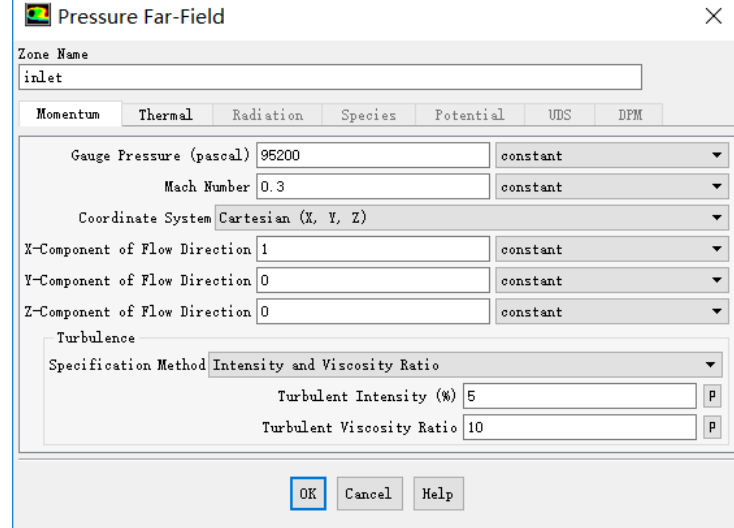

Figure 17. Partial incoming flow parameters

After setting the reference-values according to the above parameters, we set two monitors to draw the lift coefficient curve and the drag coefficient curve in the simulation calculation.

After 1667 iterations, the monitoring curves of lift coefficient and drag coefficient were obtained:

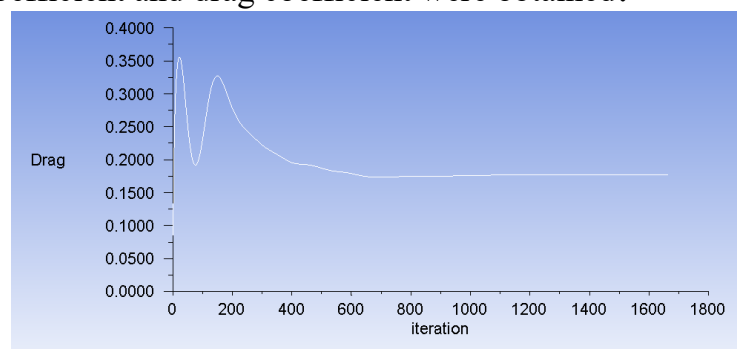

Figure 18. Drag coefficient curve

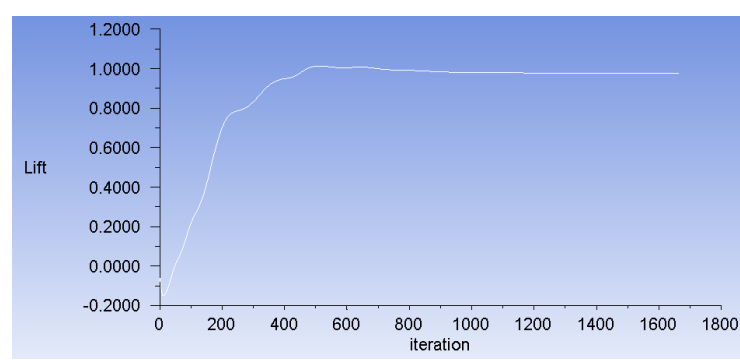

Figure 19. lift coefficient curve

It can be seen that after about 800 iterations, the lift coefficient curve and the drag coefficient curve have been basically stable. Taking the average of the last 200 sets of data, the full-aircraft lift coefficient is 0.978 , the full-aircraft drag coefficient is 0.177 , and the full-aircraft lift-drag ratio can reach 5.53. The value of thrust-weight ratio in horizontal flight is 0.181 , compared to traditional tilting rotor aircraft like MV22, it's thrust-weight ratio in horizontal flight is about $0.33^{[10]}$, the aerodynamic efficiency of this design is MV22's $182.5 \%$. Some corners of the design can be further smoothed, it may could reduce drag coefficient, thus promote lift-drag ratio.

\section{Conclusion}

In this article, a tilting rotor cargo UAV based on variable shaft Angle gear drive is roughly designed. The design can reduce the weight of the rotor tilting mechanism thus reduce the weight of the aircraft. And 
the transmission mechanism is simple, plus the failure ratio is lower than the design of tilting the whole engine nacelle. In which the engine exhaust gas always sprayed backwards in this design, so this aircraft has better site adaptability and it's more beneficial to the safety of the ground staff. The design of this paper is quite theoretical. It covers only the design of its aerodynamic shape, the design of the key parts and its mechanical structure, Some details need to be further optimized and improved. However, this paper provided a preliminary design scheme of an advanced tilt rotor aircraft, which could provide certain reference significance.

\section{References}

1. Xu Min. The summarize of development and key technologies of tilt rotor aircraft. J. Helicopter Technique. 2 (2003)

2. Airfoil Investigation Database DB/OL. http://www.airfoildb.com/, June 01, 2016 00:41

3. Zhang Xijin. Aircraft design manual 6 aerodynamic design. M. Beijing: Aeronautical Industry Press, 2002.21 198

4. Cheng Bushi. Aircraft design manual 5 overall design of civil aircraft. M. Beijing: Aeronautical Industry Press, 2005.109 220

5. Cheng Junzhang. Aircraft design manual 8 Weight balance and control. M. Beijing: Aeronautical Industry Press, 1999.103 326

6. G.E.Juola, H.R.Welge, D.N.Smyth. Analytical and Experimental Study of a Complex 3-D Inlet for Turboprop Applications. J.AIAA 84-2203,1984

7. Xu Hong Li.Analytical Design of Turboprop SDuct Intake. D.Nanjing University of Aeronautics and Astronautics.2015

8. Chen Songlu. Aircraft design manual 13 System design of power plant. M. Beijing: Aeronautical Industry Press, 2006.105 106

9. Xiao Shenyu,Shi Xiaoying.Study on the key problems of aircraft landing gear design. J. Scientific and technological innovation and Application. 7(2017)

10. Zhang Guanglin.A record of the development of the US Navy MV-22 "Osprey" tilt rotor aircraft. J.Modern Ships, 2005, (5B): 12-21 